\title{
Obesidade, aptidão cardiorrespiratória, atividade física e tempo de tela em escolares da zona urbana e rural de Santa Cruz do Sul-RS
}

\author{
Obesity, cardiorespiratory fitness, physical activity and screen time in \\ school children from urban and rural area of Santa Cruz do Sul, Brazil
}

Cézane Priscila Reuter, ${ }^{1}$ Miria Suzana Burgos, ${ }^{1}$ Cássio Virgílio Pritsch, ${ }^{1}$ Priscila Tatiana da Silva, ${ }^{1}$ Kelin Cristina Marques, ${ }^{1}$ Sonimar de Souza, ${ }^{1}$ Luiza Pasa, ${ }^{1}$ Rafaela da Silva, ${ }^{1}$ Rodrigo Muradás. ${ }^{1}$

'Universidade de Santa Cruz do Sul (UNISC), Santa Cruz do Sul, RS, Brasil.

Recebido em: março 2015 / Aceito em: abril 2015

cezanereuter@unisc.br

\section{RESUMO}

Objetivo: verificar possíveis diferenças no perfil de obesidade, aptidão cardiorrespiratória (APCR), prática de atividade física e tempo de tela em escolares. Método: os sujeitos do presente estudo são 658 escolares de 7 a 17 anos, sendo 307 do sexo masculino, pertencentes a 12 escolas (municipais e estaduais), da zona urbana e rural de Santa Cruz do Sul-RS. A obesidade foi avaliada através do índice de massa corporal (IMC), circunferência da cintura e percentual de gordura. A APCR foi avaliada através do teste de corrida/caminhada de 9 minutos. A prática de atividade física e tempo de tela foram obtidas por meio de questionários. Resultados: Os resultados demonstraram que não há associação entre o local de residência com os indicadores de obesidade. Com relação ao IMC, a prevalência de sobrepeso e obesidade encontrada foi de 29,5\%. Escolares da zona urbana apresentam prevalência $9 \%$ superior de baixos níveis de APCR; além disso, também é mais prevalente entre esses escolares passar mais de duas horas em frente à tela da TV, computador e videogame (RP: 1,07; $p=0,008)$. Considerações finais: Conclui-se que escolares da zona urbana possuem prevalência superior para baixos níveis de APCR e para o tempo de tela superior a duas horas diárias. Não foram observadas diferenças significativas com relação aos indicadores de obesidade, na comparação entre as regiões. É importante ressaltar também que, de forma geral, os escolares avaliados apresentam elevado percentual de sobrepeso e obesidade, baixos níveis de aptidão cardiorrespiratória, inatividade física e despendem mais de duas horas diárias em frente às telas da TV, do computador e do videogame.

Palavras-chave: Estilo de Vida; Aptidão Cardiorrespiratória; Obesidade; Crianças; Adolescentes.

\section{ABTRACT}

Objective: this study aims to determine possible differences in obesity profile, cardiorespiratory fitness (CRF), physical activity practice and screen time in school children. Method: the subjects of this study are 658 school students (307 boys) from 7 to 17 years belonging to 12 schools (state and local), from urban and rural area of Santa Cruz do Sul in state of Rio Grande do Sul. Obesity was assessed by using the body mass index (BMI), waist circumference and body fat percentage. The CRF was assessed through the 9 minutes walk test. The practice of physical activity and screen time were obtained through questionnaires. Results: results showed no association between place of residence and obesity indicators. Regarding $B M I$, the prevalence of overweight and obesity found was $29.5 \%$. Students in the urban area show $9 \%$ higher prevalence of CRF low levels. Furthermore, it is also more prevalent among these school students to spend more than two hours in front of the TV, computer and video game screen (RP: 1.07; $p=0.008$ ). Final considerations: we conclude that school children from the urban area have higher prevalence for low levels of CRF and screen time over to two hours daily. No significant differences were observed in relation to obesity indicators, comparing the regions. It is also important to note that in general the evaluated schoolchildren showed high rates of overweight and obesity, low levels of cardiorespiratory fitness, physical inactivity and expend more than two hours daily in front of TV, computer and video game screen.

Keywords: Life Style; Cardiorespiratory Fitness; Obesity; Children; Teenager. 


\section{INTRODUÇÃO}

A expressiva mudança de cultura das crianças que residem no meio urbano, no século $\mathrm{XXI}$, proporcionou o acesso à tecnologia e o não incentivo à prática de atividades recreativas, lúdicas, cognitivas, entre outras. Os jogos eletrônicos, fruto do avanço tecnológico, contribuíram para mudanças de hábitos, diminuindo a prática de exercícios físicos ${ }^{1}$. Além disso, assistir excessivamente televisão durante a infância e adolescência tem sido associado aos indicadores de saúde desfavoráveis, como o desenvolvimento de baixa aptidão física, colesterol elevado e obesidade ${ }^{2}$.

Atualmente, um dos maiores problemas mundiais é a obesidade, a qual apresenta aumento considerável, tanto na zona rural, como na zona urbana, sendo encontrada em todas as faixas etárias e causando modificações físicas e psicológicas ${ }^{3,4}$. Em escolares obesos, o início da puberdade poderá ser precoce, o que fará com que seu crescimento cesse antes do previsto, diminuindo sua altura final, pois suas cartilagens de crescimento terão seu fechamento adiantado ${ }^{5}$.

A gordura na região abdominal é caracterizada como obesidade abdominal visceral, sendo este um grande fator para complicações cardiovasculares e distúrbio na homeostase glicose-insulina, mais do que a obesidade generalizada. A obesidade também está associada a dislipidemias, hipertensão, fibrinólise, aceleração da progressão da aterosclerose e fatores psicossociais. A síndrome metabólica também é um dos malefícios que a obesidade provoca ${ }^{6}$.

As doenças cardiovasculares, câncer, diabetes mellitus e hipertensão arterial são doenças não transmissíveis que caracterizam um grupo de pessoas que apresentam uma forma prolongada no período de latência, complicações irreversíveis que acarretam graus de incapacidade ou óbito, que está ocupando cada vez mais o perfil de morbimortalidade nas populações latino americanas ${ }^{7}$. Segundo o Banco Mundial ${ }^{8}$, um dos maiores responsáveis por mortes prematuras são as doenças crônicas não transmissíveis, tendo uma taxa de 5 e 9 vezes maior do que as doenças transmissíveis, responsáveis por uma taxa de 10 e 5 vezes maiores de incapacidade, em ambos os sexos.

A nível mundial, pesquisas verificaram o excesso de peso que tem aumentado consideravelmente. $\mathrm{Na}$ China, em Taiwan, a prevalência do excesso de peso na fase da infância praticamente dobrou entre 1986 (13\%) e $2009(27,7 \%)^{9}$. Nos Estados Unidos, 31,8\% das criancas de 2 a 19 anos de idade apresentam sobrepeso ou obesidade $^{10}$. No Brasil, a prevalência de obesidade vem aumentando nas últimas décadas, afetando todas as regiões e classes sociais do país. Dados da população sobre o estado nutricional infantil brasileiro ressaltaram que a prevalência de excesso de peso em jovens, com idades entre 5 e 9 anos na região Norte e Nordeste, é de $25 \%$ a $30 \%$. Nas regiões Sul, Sudeste e Centro-Oeste, o percentual variou entre 32 a $40 \%^{11}$. No município de Santa Cruz do Sul-RS, esse percentual é de $27,1 \%$ para os meninos e $26,4 \%$ para as meninas ${ }^{12}$.

Dessa forma, reconhecer a obesidade infantil precocemente é de suma importância, pois quanto mais cedo for realizada uma intervenção, preferencialmente antes dos 10 anos de idade, reduzem-se as chances dessa doença persistir na vida adulta. Com isso, devem-se influenciar hábitos saudáveis desde a primeira infância, conscientizando familiares sobre a importância da alimentação com modificações no balanço calórico e realizando atividade física moderada. As instituições de ensino devem sempre estar atentas a esse problema, servindo de apoio às famílias, orientando e conscientizando sobre todos os problemas decorrentes da obesidade ${ }^{13}$, a qual também pode trazer distúrbios psicológicos caracterizados por mudanças nos padrões comportamentais das crianças, as quais podem vir a sofrer de depressão, baixa estima e ser vítima de bullying, tanto na escola quanto fora deste espaço e, com isso, buscando o isolamento da sociedade ${ }^{14}$.

Nessa perspectiva, o presente estudo tem como objetivo verificar possíveis diferenças no perfil de obesidade, aptidão cardiorrespiratória, prática de atividade física e tempo de tela em escolares da zona urbana e rural do município de Santa Cruz do Sul-RS.

\section{MÉTODO}

Estudo transversal composto por uma amostra de 658 escolares de 7 a 17 anos, sendo 307 do sexo masculino, pertencentes a 12 escolas (municipais e estaduais), da zona urbana e rural de Santa Cruz do Sul-RS, o qual é recorte de uma pesquisa mais ampla, denominada "Avaliação de indicadores bioquímicos de saúde de escolares, usando espectroscopia no infravermelho, polimorfismos, saúde bucal e fatores relacionados ao estilo de vida: um estudo em Santa Cruz do Sul - Fase II", desenvolvida na Universidade de Santa Cruz do Sul - RS (UNISC). A referida pesquisa foi encaminhada previamente à coleta de dados ao Comitê de Ética em Pesquisa com Seres Humanos da UNISC, obtendo aprovação sob protocolo número 2959-11. Todos os pais ou responsáveis autorizaram a participação dos escolares no estudo, mediante assinatura do termo de consentimento livre e esclarecido.

Os indicadores de obesidade avaliados foram: índice de massa corporal (IMC), circunferência da cintura (CC) e percentual de gordura corporal (\%G). O IMC foi calculado através das medidas de peso e estatura. Posteriormente, os dados foram classificados de acordo com os pontos de corte do Centers for Disease Control and Prevention/National Center for Health Statistics ${ }^{15}$, considerando baixo peso $(<p 5)$, normal ( $\geq p 5$ e $<p 85)$, sobrepeso ( $p 85$ e $<p 95$ ) e obesidade ( $\geq p 95$ ), os quais foram agrupados em "baixo peso/normal" e "sobrepeso/ obesidade". A CC foi avaliada através de fita métrica inelástica, tendo como referência a parte mais estreita do tronco entre as costelas e a crista ilíaca e o quadril no nível do trocanter maior. Posteriormente, os dados foram classificados de acordo com os pontos de corte de Taylor et al. ${ }^{16}$, considerando circunferência normal (percentil $\leq 80$ ) e obesidade (percentil >80), considerando sexo e idade. Para o cálculo do \%G, utilizou-se as dobras tricipital e subescapular, através da equação de Slaugther et al. ${ }^{17}$. Para a classificação, utilizou-se os dados de Lonman ${ }^{18}$, o qual divide o \%G em cinco categorias: muito baixo, baixo, ótimo, moderadamente alto, alto e muito alto. As três primeiras categorias foram consideradas como "sem risco" e as três últimas como "risco". 
A aptidão cardiorrespiratória (APCR) foi avaliada através da realização do teste de corrida/caminhada de 9 minutos $^{18}$, na pista atlética da UNISC. Os escolares foram orientados ao uso de roupa leve e tênis. Os resultados obtidos, em metros percorridos, foram registrados e posteriormente classificados de acordo com os pontos de corte para a população infanto-juvenil brasileira, definidos pelo PROESP-BR ${ }^{19}$, de acordo com sexo e idade.

A prática de atividade física (sim ou não) e o tempo de tela (TV, computador e videogame) foram avaliados por meio de questionário autorreferido pelo escolar, utilizado na pesquisa de Burgos et al. ${ }^{20}$ e adaptado de Barros e Nahas ${ }^{21}$. O tempo de tela foi registrado em horas e posteriormente classificado de acordo com os critérios estabelecidos pela Academia Americana de Pediatria ${ }^{22}$ em: 1) pouco tempo em frente à tela $(<2$ horas diárias) e 2) muito tempo em frente à tela ( $\geq 2$ horas diárias).

A análise estatística dos dados foi realizada no programa SPSS v. 20.0 (IBM, Armonk, NY, EUA). Foi empregada a estatística descritiva para caracterização da amostra, através de frequência e percentual. A associação das variáveis desfecho (indicadores de obesidade, aptidão cardiorrespiratória, atividade física e tempo de tela) com a variável preditora (zona de moradia), foi testada pela regressão de Poisson, através dos valores de razão de prevalência, considerando o intervalo de confiança de 95\% e nível de significância para $p<0,05$.

\section{RESULTADOS}

As características descritivas demonstram que é elevado o percentual de escolares com obesidade, principalmente com percentual de gordura elevado $(45,1 \%)$. Além disso, ressalta-se que $52,0 \%$ possuem baixos níveis de aptidão cardiorrespiratória e 55,5\% despendem 2 horas ou mais por dia em frente à TV, computador ou videogame (Tabela 1).

A tabela 2 demonstra que não existe associação entre os indicadores de obesidade (IMC, CC e \%G) com a região de moradia do escolar.

Por outro lado, entre os escolares da zona urbana, a prevalência de baixos níveis de APCR é $9 \%$ maior, bem como é superior a prevalência de tempo de tela por duas horas diárias ou mais (RP: 1,07; $p=0,008$ ) (Tabela 3).
Tabela 1 - Características descritivas dos sujeitos.

\begin{tabular}{ll}
\hline & $\mathbf{n}(\%)$ \\
\hline Região de moradia & $330(50,2)$ \\
$\quad$ Rural & $328(49,8)$ \\
Urbana & \\
IMC & $464(70,5)$ \\
$\quad$ Baixo peso/normal & $194(29,5)$ \\
$\quad$ Sobrepeso/obesidade & \\
CC & $538(81,8)$ \\
$\quad$ Normal & $120(18,2)$ \\
Elevada & \\
\%G & $361(54,9)$ \\
Sem risco & $297(45,1)$ \\
Risco & \\
APCR & $316(48,0)$ \\
$\quad$ Normal & $342(52,0)$ \\
Baixos níveis & \\
Atividade física & $403(61,2)$ \\
Sim & $255(38,8)$ \\
Não & $293(44,5)$ \\
Tempo de tela & $365(55,5)$ \\
Até 2 horas diárias & \\
2 horas ou mais diárias &
\end{tabular}

IMC: índice de massa corporal; CC: circunferência da cintura; \%G: percentual de gordura corporal; APCR: aptidão cardiorrespiratória.

\section{DISCUSSÃO}

Comparando os valores médios de IMC, o presente estudo não demonstrou associação entre o local de residência com os indicadores de obesidade, sendo que a prevalência de excesso de peso (sobrepeso e obesidade) encontrada nos escolares avaliados foi de $29,5 \%$, de acordo com o IMC. Resultado similar ao encontrado em estudo realizado no município de Morro Reuter e Dois Irmãos (RS), no qual $24,4 \%$ dos alunos apresentaram sobrepeso e obesidade ${ }^{23}$. No entanto, outra pesquisa realizada em Santa Cruz do Sul-RS, analisando apenas a prevalência de sobrepeso e obesidade entre as regiões, demonstrou percentual superior para a zona urbana ${ }^{24}$.

No presente estudo, também não foram observadas diferenças na comparação da obesidade abdominal, avaliada pela CC, entre as regiões; porém, o percentual encontrado foi elevado $(18,2 \%)$. Estudo realizado na

Tabela 2 - Associação entre indicadores de obesidade e região de moradia.

\begin{tabular}{lccccc}
\hline & IMC & p & CC & p & \%G \\
\hline Região & RP (IC 95\%) & & RP (IC 95\%) & & RP (IC 95\%) \\
Rural & 1 & 0,187 & 1 & 0,714 & 1 \\
Urbana & $1,04(0,98-1,09)$ & & $1,01(0,96-1,06)$ & & $1,05(0,99-1,10)$ \\
\hline
\end{tabular}

IMC: índice de massa corporal; CC: circunferência da cintura; \%G: percentual de gordura corporal.

Tabela 3 - Associação entre indicadores de atividade física e sedentarismo com a região de moradia.

\begin{tabular}{lccccc}
\hline & APCR & $\mathbf{p}$ & Atividade Física & p & Tempo de Tela \\
\hline Região & RP (IC 95\%) & & RP (IC 95\%) & p & RP (IC 95\%) \\
Rural & 1 & $<0,001$ & 1 & 0,714 & 1 \\
Urbana & $1,09(1,04-1,15)$ & & $1,02(0,97-1,06)$ & 0,008 \\
\hline
\end{tabular}

APCR: aptidão cardiorrespiratória. 
China demonstrou que a obesidade abdominal é superior na zona urbana, tanto entre os meninos $(19,92 \%$ para zona urbana e $11,94 \%$ para zona rural), quanto nas meninas $(8,43 \%$ para zona urbana e $5,85 \%$ para zona rural), evidenciando uma relação entre o local de moradia com a obesidade abdominal ${ }^{25}$. Outro estudo, também realizado na China, mostra um aumento nas taxas de crianças obesas entre 1985 e 2010, passando de 0,1\% para $5 \%$, sendo que os que residem na zona urbana apresentam maiores índices de obesidade também ${ }^{26}$.

Com relação à prática de atividade física e sedentarismo, os escolares da zona urbana apresentam prevalência 9\% superior de baixos níveis de APCR; além disso, também é mais prevalente entre esses escolares passar mais de duas horas em frente à tela da TV, computador e videogame (RP: 1,$07 ; p=0,008$ ). Estudo longitudinal realizado em Dunedim, Nova Zelândia, avaliou crianças e adolescentes de 5 a 15 anos e os acompanhou até os 26 anos de idade, para investigar associações entre tempo de tela, IMC e APCR. Constatou-se que assistir televisão na infância e adolescência está associada com excesso de peso, baixos níveis de APCR, colesterol sérico elevado e tabagismo na idade adulta ${ }^{2}$. Na Grécia, um estudo demostrou que, apesar dos índices de crianças obesas na zona rural ter aumentado nos últimos anos, os testes de aptidão física ainda são melhores se comparados aos de crianças de zona urbana ${ }^{27}$.

Entre crianças e adolescentes iranianos, 33,4\% dos que residem em zona rural assistem TV/vídeo mais de duas horas em seu tempo de lazer, contra $53 \%$ dos residentes em zona urbana. Do mesmo modo, 6,3\% e $10,9 \%$ dos alunos usaram o computador, mais de 2 horas, em seu tempo de lazer, havendo um tempo de tela muito elevado em ambas as localidades ${ }^{28}$. Esse elevado número de horas despendido em tempo de tela pelas crianças em nosso estudo sugere que os escolares avaliados têm gasto menos tempo na prática de atividade física. Níveis de atividade física, durante a infância e adolescência, de forma insuficiente, estão frequentemente associados ao acúmulo de gordura corporal em excesso, aumento de alterações no perfil lipídico sanguíneo, elevação da pressão arterial ${ }^{29}$, contato com comportamentos que forneçam risco à saúde e chances maiores de ser um indivíduo sedentário na fase adulta ${ }^{30}$. Haskell et al. ${ }^{31}$ ressaltam que o sedentarismo (inatividade física) está diretamente vinculado com o aparecimento de doenças, como: hipertensão, acidente vascular encefálico, osteoporose, diabetes, câncer, obesidade, depressão entre outras.

Os resultados do presente estudo apontam, assim, uma urgente necessidade de construção de estratégias para reduzir esses fatores de riscos silenciosos. De acordo com estudo realizado por Rivera et al. ${ }^{32}$, as estratégias de prevenção de saúde para essas faixas etárias devem envolver maior oferta de atividade físicas e redução do tempo despendido em atividades sedentárias. Além disso, estudos recentes ${ }^{33-35}$ ressaltam que o meio sociocultural em que a criança está inserida (escola), os pais e a comunidade devem oferecer suporte adequado e suficiente para que a criança e adolescente possam moldar novos hábitos saudáveis. Nesse sentido, a implantação de programas de intervenção com exercícios físicos e reeducação de hábitos alimentares, bem como sobre o uso da televisão, são importante estratégias para redução de fatores de risco $^{36,37}$.

\section{CONSIDERACÕ̃ES FINAIS}

Conclui-se que escolares da zona urbana possuem prevalência superior para baixos níveis de aptidão cardiorrespiratória e para o tempo de tela superior a duas horas diárias. Não foram observadas diferenças significativas com relação aos indicadores de obesidade, na comparação entre as regiões. É importante ressaltar também que, de forma geral, os escolares avaliados apresentam elevado percentual de sobrepeso e obesidade, baixos níveis de aptidão cardiorrespiratória, inatividade física e despendem mais de duas horas diárias em frente às telas da TV, do computador e do videogame.

\section{REFERÊNCIAS}

1. Terres NG, Pinheiro RT, Horta BL Pinheiro KAT, Horta LL. Prevalência e fatores associados ao sobrepeso e à obesidade em adolescentes. Rev Saude Publica. 2006;40 (4):627-33.

2. Hancox RJ, Milne BJ, Poulton R. Association between child and television viewing and adult health: a longitudinal birth cohort study. Lancet. 2004;364(9430):257-62.

3. Ribeiro AS, Filho CAC, Cunha LA, Netto JES, Oishi LM, Alves PMM. Prevalência de sobrepeso e obesidade em crianças de $3^{\mathrm{a}}$ e $4^{\mathrm{a}}$ série do ensino fundamental. Rev Colloquium Vitae. $2011 ; 3(1): 1-5$

4. Vázquez-Navas F, Manzo NTG, Rodríguez CF, Rodríguez EM. Association between family structure, maternal education level and maternal employment with sedentary lifestyle in primary school-age children. J Pediatr (Rio J). 2012;89(2):145-50.

5. Hammer LD. Pediatria Ambulatorial. Porto Alegre: Artes Médicas, 1992.

6. Martins IS, et al. Doenças cardiovasculares ateroscleróticas, dislipidemias, hipertensão, obesidade e diabetes melito em população da área metropolitana da região sudeste do Brasil. I- Metodologia da pesquisa. Rev Saude Publica. 1993;27(4):250-61.

7. Duncan BB, Schmidt MI, Polanczyk C.A, Homrich CS, Rosa RS, Achutti AC. Fatores de risco para doenças nãotransmissíveis em áreas metropolitanas na região sul do Brasil: prevalência e simultaneidade. Rev Saude Publica. 1993;27(1):24.

8. Banco Mundial - Human Development. Toward a Healthy and Harmonious Life in China: Stemming the Rising Tide of Non-Communicable Disease. Disponível em http://www. worldbank.org/content/dam/Worldbank/document/NCD report en.pdf. Acesso em 02 abr. 2015.

9. Chen LJ, Fox KR, Haase A, Wang JM. Obesity, fitness and health in Taiwanese children and adolescents. Eur $\mathrm{J}$ of Clin Nutr. 2006;60(12):1367-75.

10. Ogden CL, Carroll MD, Kit B.K, Flegal KM. Prevalence of obesity and trends in body mass index among US children and adolescent. JAMA. 2012;307(5):483-90.

11. Friedrich RR, Schuch I, Wagner M.B. Effect of interventions on the body mass index of school-age students. Rev Saude Publica. 2012;46(3):551-60.

12. Burgos MS, Reuter CP, Burgos LT, Pohl HH, Pauli LTS, Horta JÁ, Reckziegel MB. Franke SIR, Prá D, Camargo M. Uma 
análise entre índices pressóricos, obesidade e capacidade cardiorrespiratória em escolares. Arq Bras Cardiol, 2010;94(6):788-93.

13. Alves, Bianca da Silva. Obesidade na infância: critérios diagnósticos e impacto no rendimento escola. Universidade Federal Rio Grande do Sul. Faculdade de Medicina. Programa de Pós-Graduação em Ciências Médicas: Endocrinologia. Porto Alegre, 2007.

14. Silva GAP, da. Balaban, G, Motta, ME, da A. Prevalência de sobrepeso e obesidade em crianças e adolescentes de diferentes condições socioeconômicas. Rev Bras Saúde Matern Infant. 2005;5(1):53-59.

15. CDC. Centers for Disease Control and Prevention/National Center for Health Statistics. CDC Growth Charts: United States. 2000. Disponível em: <http://www.cdc.gov/ growthcharts $>$. Acesso em: 10 maio 2015

16. Taylor R W, et al. Evaluation of waist circumference, waistto-hip ratio, and the conicity index as screening tools for high trunk fat mass, as measured by dual-energy $\mathrm{X}$-ray absorptiometry, in children aged 3-19 y. Am J Clin Nutr. 2000;72(2):490-495.

17. Slaughter $\mathrm{MH}$, et al. Skinfold equations for estimation of body fatness in children and youth. Hum Biol, 1988;60(5):709-723.

18. Lohman Timothy $\mathrm{G}$. The use of skinfold to estimate body fatness on children and youth. Joperd, 1987;58(9):98-102.

19. Projeto Esporte Brasil. Manual. 2009. Disponível em: <http:// www.proesp.ufrgs.br>. Acesso em: 10 maio 2015.

20. Burgos MS. et al. Saúde dos escolares- Fase III. Avaliação de indicadores bioquímicos, genéticos, hematológicos, imunológicos, posturais, somatomotores, saúde bucal, fatores de risco às doenças cardiovasculares e estilo de vida de escolares: estudo em Santa Cruz do Sul-RS (Projeto de Pesquisa). Santa Cruz do Sul: UNISC, 2014.

21. Barros MVG, Nahas MV. Medidas da atividade física: teoria e aplicação em diversos grupos populacionais. Londrina: Midiograf, 2003.

22. AAP. American Academy of Pediatrics. Children, adolescents, and television. Pediatrics, 2001;107(2):423-426.

23. Triches R M, Giugliani, E. R. J. Obesidade, práticas alimentares e conhecimentos de nutrição em escolares. Rev Saude Publica. 2005;39(4):541-7.

24. Garibaldi F, Tornquist D, Tornquist L, Burgos MS. Prevalência de sobrepeso e obesidade em escolares de Santa cruz do Sul: um comparativo entre zona urbana e rural. Lecturas,
Educación Física y Deportes. 2014;18,189.

25. Zhang YX, Zhao JS, Chu ZH, Tan H. L. Prevalence and regional disparities in abdominal obesity among children and adolescents is Shandong, China, surveyed in 2010. Ann Nutr Metab. 2014;64(2):137-43.

26. Song Y, Ma J, Wang HJ, Wang Z, Hu P, Zhang B, Agard A. Secular trends of obesity prevalence in Chinese children from 1985 to 2010: Urban - rural disparity. Obesity. 2015;23(2):448-53.

27. Tambalis KD, Panagotakos DB, Sidossis LS. Greek children living in rural áreas are heavi er but fitter compared to their urban counterparts: a comparative, times-series (1997-2008) analysis. J Rural Health. 2011;27(3)270-7.

28. Jari M, Qorbani M, Motlagh ME, Heshmat R, Ardalan G, Kelishadi RA. Nationwide Survey on the Daily Screen Time of Iranian Children and Adolescents: The Caspian - IV Study. Int J Prev Med. 2014;5(2):224-9.

29. Duarte JA, Ribeiro JC, Oliveira J, Mota J. Relação entre níveis de atividade física e valores de colesterolemia em crianças e adolescentes. Rev Bras Saúde Matern Infant. 2004;4(2):185-92.

30. Azevedo MR, Araújo CL, Silva CM, Hallal PC. Tracking of physical activity from adolescence to adulthood: a populationbased study. Rev Saude Publica. 2007;41(1):69-75.

31. Haskell William L. et al. Physical Activity and Public Health: Updated Recommendation for Adults from the American College of Sports Medicine and the American Heart Association. Med Sci Sports Exerc. 2007;39(8):1423-34.

32. Rivera IR et al. Atividade física, horas de assistência à TV e composição corporal em crianças e adolescentes. Arq Bras Cardiol. 2010; 95(2): 159-165.

33. Camargo APPM et al. A não percepção da obesidade pode ser um obstáculo no papel das mães de cuidar de seus filhos. Cien Saude Colet. 2013;18(2):323-33.

34. Bergmann, M. L. A. et al. Colesterol total e fatores associados: estudo de base escolar no sul do Brasil. Arq Bras Cardiol. 2011;97(1):17-25.

35. Cugnetto ML et al. Lifestyle factors, body mass index, and lipid profile in adolescents. J Pediatr Psychol. 2008;33(7):761-71.

36. Soares LD, Petroski ED. Prevalência, fatores etiológicos e tratamento da obesidade infantil. Rev Bras Cineantropom Desempenho Hum. 2003;5(1):63-74.

37. Silva VL. et al. Avaliação de programa de prevenção de obesidade em adolescentes de escolas públicas. Rev Saude Publica. 2011;45(1):59-68. 